\title{
Application of ROMA (representational oligonucleotide microarray analysis) to patients with cytogenetic rearrangements
}

Vaidehi Jobanputra, $P h D^{1}$, Jonathan Sebat, $P h D^{4}$, Jennifer Troge, $P h D^{4}$, Wendy Chung, MD, $P h D^{3}$, Kwame Anyane-Yeboa, $\mathrm{MD}^{2}$, Michael Wigler, $\mathrm{PhD}^{3,4}$ and Dorothy Warburton, $P h D^{3}$

\begin{abstract}
Purpose: To demonstrate the accuracy and sensitivity of Representational Oligonucleotide Microarray Analysis (ROMA) to describe copy number changes in patients with chromosomal abnormalities. Methods: ROMA was performed using Bglll digested DNA from two cases with cytogenetically detected deletions and one case with an unbalanced terminal rearrangement detected only by subtelomeric FISH. Hybridization was to an 85,000-probe oligonucleotide microarray, providing an average resolution of $35 \mathrm{~kb}$. FISH was used to confirm some of the ROMA findings. Results: By ROMA, a del(13)(q14.3q21.2) was shown to be noncontiguous, with deletions extending from 53.08 to $61.40 \mathrm{Mb}$ and from 72.88 to $74.83 \mathrm{Mb}$. The 10-Mb deletion contained only six known genes. FISH confirmed the noncontiguous nature of the deletion, as well as a small amplification in $6 q$ that was also found in the patient's mother. A del(4)(q12q21.2) was found by ROMA to be $23 \mathrm{Mb}$ in length, from 58.8 to $81.9 \mathrm{Mb}$ on chromosome 4, in agreement with the cytogenetically assigned breakpoints. ROMA showed that an unbalanced "subtelomeric" rearrangement involved a 6-Mb deletion of 22q and an 8-Mb duplication of 16q. Conclusions: ROMA can define cytogenetic aberrations with extraordinary precision. Unexpected findings included the interrupted nature of the deletion in 13q and the large size of the imbalances in the "subtelomeric" rearrangement. Together with the information from the human genome sequence and proteomics, the ability to define rearrangements with "ultra-high" resolution will improve the ability to provide accurate prognosis both prenatally and postnatally to parents of offspring with chromosomal aberrations. Genet Med 2005:7(2):111-118.
\end{abstract}

Key Words: cytogenetics, deletion, microarray, oligonucleotide, ROMA

The resolution of cytogenetic analysis has been continually improved through the introduction of new techniques, such as high-resolution banding, FISH analysis for microdeletions and subtelomeric rearrangements, multichromosome painting by SKY or M-FISH, and chromosomal comparative genomic hybridization (CGH). Most recently, $\mathrm{CGH}$ on genomic microarrays has permitted high-resolution chromosome scanning to define copy number variation in the genome. The usual methods involve BAC micoarrays, which can be selected to provide varying levels of resolution. A complete tiling microarray across the complete genome has been reported, ${ }^{1}$ with a theoretical resolution of 100 to $200 \mathrm{~Kb}$, but clinical application of whole genome scanning has been largely limited to arrays with $\geq 1-\mathrm{Mb}$ resolution. One recent study ${ }^{2}$ reports de novo chro-

From the ${ }^{1}$ Department of Obstetrics and Gynecology, ${ }^{2}$ Pediatrics, ${ }^{3}$ Genetics and Development and Pediatrics, Columbia University Medical Center, New York, NY; and ${ }^{4}$ Cold Spring Harbor Laboratory of Biology, Cold Spring, NY.

Dorothy Warburton, Genetics Laboratory, The Children's Hospital of New York, Rm CHC 406, 3959 Broadway, New York, NY 10032.

Received: August 16, 2004.

Accepted: October 13, 2004

DOI: 10.1097/01.GIM.0000153661.11110.FB mosomal deletions or duplications in 7 of 50 patients with learning disability and dysmorphic features, suggesting that such rearrangements may be quite frequent as causes of congenital abnormalities.

Representational Oligonucleotide Microarray Analysis (ROMA) is a new approach that allows very high resolution scanning for copy number variation across the whole genome. ${ }^{3}$ In brief, this method involves the following: (1) digestion of the subject's DNA with an enzyme such as BglII, followed by amplification by adaptor-mediated PCR, resulting in a "representation" of the genome; (2) design of a microarray using probes hybridizing with short 200 to $1200 \mathrm{bp}$ restriction fragments and preselected for good hybridization performance. ${ }^{4}$ The microarrays used in this study consist of 85,000 probes (85K), with a resolution of about $35 \mathrm{~kb}$; (3) CGH to the microarray of representations from test and control DNA; and (4) statistical analysis using a Hidden Markov model ${ }^{5}$ to determine segmental deletions or duplications.

To date, ROMA has been used to define copy number changes in tumors ${ }^{3}$ and to reveal the extent of the large-scale copy number polymorphism in the human genome. ${ }^{5}$ In this report, we present the first use of ROMA on three children with known constitutional chromosome abnormalities, which 
serves not only to validate the method, but also to illustrate the extraordinary precision with which we can now define chromosomal imbalances. Not only did ROMA reveal unexpected complexity, but also the precise definition of the abnormalities was useful in interpreting the clinical findings and providing information to the parents.

\section{PATIENT MATERIAL AND METHODS}

\section{Clinical information}

\section{Patient 1}

Patient 1 is the third child of a G4P4 32-year-old mother and a 33-year-old father. The proband's pregnancy and delivery were uncomplicated, and his birth weight was $7 \mathrm{lb} 9 \mathrm{oz}$. He was initially evaluated at the age of 3 months because of failure to thrive, hypotonia, and associated feeding difficulties. MRI of brain with spectroscopy and echocardiogram were normal. Renal ultrasound demonstrated a small left kidney. Extensive metabolic and genetic evaluations revealed only the karyotypic abnormality discussed below.

The child was seen at Columbia University Medical Center for the first time at age 2 years and 2 months. At that time, the height and weight were in the 25 th percentile. The mother reported delayed major motor milestones, including rolling over at 4 months, sitting without support at 8 months, and walking at 22 months. He had been receiving regular physical therapy for delay in gross motor skills. He spoke in short sentences and understood complex commands. There were no facial dysmorphisms besides slightly cupped ears and no medical problems except for severe eczema.

At three years, development status is normal, except for mild delay in gross motor skills and coordination. The child converses in full sentences, retells stories, knows colors, and repeats nursery rhymes. Height and weight remain in the 25th percentile.

\section{Patient 2}

Patient 2 was a 19-month-old male at the time of initial evaluation. He was the product of a 37-week gestation, born via C-section for failure to progress to a 40 -year-old G1P0 woman. An amniocentesis performed at another institution for advanced maternal age was reported as a normal $46 \mathrm{XY}$ karyotype. Birth weight was $6 \mathrm{lb} 2 \mathrm{oz}(10 \%)$, and length was 18 inches $(<5 \%)$. His neonatal course was complicated by vomiting associated with a large anterior fontanel, which prompted a MRI that demonstrated a left caudate infarct of prenatal origin. He had persistent failure to thrive with height and weight less than the third percentiles for the first 18 months of life, associated with gastroesophageal reflux, constipation, and recurrent postprandial hypoglycemia that ultimately required gastrostomy tube placement and continuous feeds to avoid hypoglycemia. Several months after starting his continuous feeds, his weight normalized to the 25th percentile, and his length normalized to the 10th percentile. There was no evidence of hyperinsulinism, growth hormone deficiency, adre- nal insufficiency, fatty acid oxidation disorder, organic acidemia, or glycogen storage disorder. He has renal tubular acidosis and renal calculi and a history of recurrent otitis media and pneumonia. He is hypotonic and developmentally delayed, with a developmental quotient of 52 . His mildly dysmorphic features include a triangular face, delayed closing of the anterior fontanel, frontal bossing with a wide forehead, deeply set eyes, highly arched palate, and micrognathia.

\section{Patient 3}

Patient 3 was the $7 \mathrm{lb} 12 \mathrm{oz}$ product of a 38-week pregnancy and normal delivery. At 28 months, she was evaluated and was noted to have significant developmental delay, microcephaly, hypotonia, joint laxity at the wrist and fingers, contractures of the knees, and dysmorphic features including bilateral epicanthal folds, low set posteriorly rotated ears, wide upper gums and a narrow high-arched palate, widely spaced nipples, and wrinkling of the skin on the hands, palms, and soles of the feet. MRI of the brain demonstrated an absent corpus callosum, and hydronephrosis was observed on the abdominal ultrasound. At 4 years, 9 months, the patient has no verbal language and is severely developmentally delayed.

\section{Cytogenetic analysis and FISH}

Cytogenetic analysis was performed from peripheral blood samples using G-banding at the 550 level of resolution. FISH analysis for subtelomeric regions used the ToTelVysion probe set (Vysis, Inc. Downer's Grove, IL) and the method recommended by the manufacturer. SKY analysis on Patient 1 used the Applied Spectral Imaging Inc (Carlsbad, CA) system.

To validate the genome scan, we performed FISH analysis using BAC/PAC clones from the deleted, nondeleted, and amplified regions to metaphase chromosomes and interphase nuclei of Patient 1 and his parents. BAC clones mapping to the appropriate regions were selected from the UCSC Genome Browser (http://genome.ucsc.edu/) and purchased from the BacPac Resources Centre (http://www.chori.org/bacpac/). Single BAC colonies were inoculated in $\mathrm{LB} /$ chloramphenicol and the PAC colonies were inoculated in LB/kanamycin and cultured overnight, followed by DNA preparation using the Qiagen Large Construct DNA preparation procedure. One microgram of the cloned DNA was labeled using either Spectrum Red or Spectrum Green dUTP by nick translation labeling kit (Vysis, Downer's Grove, IL). For cohybridization of neighboring BAC clones, the probes were differentially labeled using either spectrum red or green. FISH was performed according to the manufacturer's protocol (Vysis, Downer's Grove, IL).

\section{Genome scan for copy number change using ROMA}

Analysis was blind to the karyotypic findings. ROMA was performed using an $85 \mathrm{k}$ chip with approximately $35-\mathrm{kb}$ resolution across the genome, as previously described. ${ }^{3}$ In brief, DNA was isolated from peripheral blood lymphocytes using a FlexiGene DNA kit (Qiagen, CA) according to the manufacturer's instructions. BglII representations were prepared from the patient DNA and the normal male reference DNA. After 
BglII digestion, adapters were ligated and the resulting product used as a template for a PCR reaction. The resulting genomic representation was hybridized to the $85 \mathrm{k}$ chip (NimbleGen Systems, Reykjavic, Iceland) and scanned by an Axon GenePix $4000 \mathrm{~A}$ scanner. Segmental changes in copy number were identified using a Hidden Markov Model predictor. ${ }^{5}$

The protocol for this study was approved by the Columbia Institutional Review Board and informed consent for the ROMA analysis was obtained from the parents of all the patients.

\section{RESULTS}

\section{Cytogenetic analysis}

\section{Patient 1}

At 3 months, the karyotype was found to have an apparently balanced translocation between the long arms of chromosome 3 and 10 (Fig. 1a), as well as an interstitial deletion of chromosome 13 with breakpoints in q14.3 and q21.2 (Fig. 1b): the complete karyotype was 46,XY,t(3;10)(q23;q11.2),del(13) (q14.3q21.2). Parental chromosomes were normal. The G-banded karyotype was later confirmed by FISH using the SKY technique, which demonstrated the known translocation between chromosome 3 and 10 but no more complex rearrangements. FISH using the LSI 13 probe (Vysis, Inc.) showed that the RB1 locus was not deleted.

\section{Patient 2}

Repeat of the karyotype at Columbia showed a large deletion of proximal chromosome 4q, 46,XY,del(4)(q12q21.2) (Fig. 2).
Parental chromosomes were normal. FISH with chromosome 4 paint did not reveal any additional abnormalities.

\section{Patient 3}

The karyotype by G-banding was normal 46,XX. FISH using the ToTelVysion probe set (Vysis, Inc) revealed an unbalanced terminal rearrangement, with an additional copy of the $16 \mathrm{q}$ subtelomeric probe on the end of the $\mathrm{q}$ arm of a small acrocentric chromosome and a deleted copy of the 22q subtelomeric probe. Parental chromosome analysis using subtelomeric probes showed that the father was a carrier of a balanced translocation: 46,XY,t(16;22)(q24.3;q13.3). The child's karyotype is thus: $46, X X, \operatorname{der}(22) t(16 ; 22)(\mathrm{q} 24.3 ; \mathrm{q} 13.3)$ pat.

\section{ROMA analysis}

All cytogenetically visible chromosomal imbalances were also detected by ROMA. These abnormal lesions were easily distinguishable from normal copy number polymorphism $(\mathrm{CNP})^{5}$ based on their size. The smallest aberration detected was approximately $2 \mathrm{Mb}$ in size and involved 68 probes, which is substantially larger than the average length of a CNP (460 $\mathrm{Kb}) .{ }^{5}$ In addition, numerous smaller intervals of copy number difference were detected, most of which are likely to be normal CNPs. With the exception of one rare CNP observed in Patient 1 , none of the smaller intervals was investigated further.

\section{Patient 1}

The cytogenetically visible interstitial deletion of $13 \mathrm{q}$ was revealed to consist of two noncontiguous deleted segments. The first region ( 221 probes) extended from 53,075,360 to
A
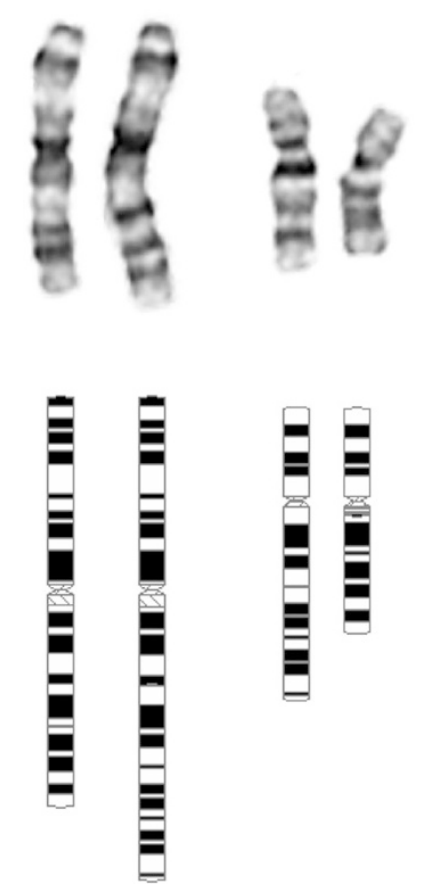

Fig. 1. Partial karyotypes from Patient 1. A, Balanced translocation $\mathrm{t}(3 ; 10)(\mathrm{q} 23 ; \mathrm{q} 11.2)$. B, Chromosome 13q deletion: $\operatorname{del}(13)(\mathrm{q} 14.3 \mathrm{q} 21.2)$.

\section{B}
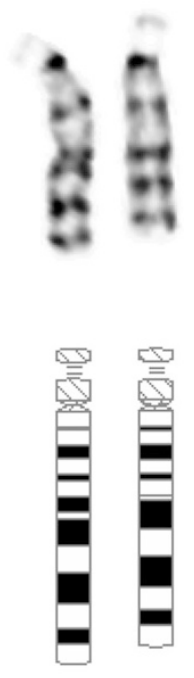
$61,396,479$ bp and the second (68 probes) from $72,882,686$ to $74,827,260$ bp (Fig. 3). No deletion or amplification was detected at either of the breakpoint regions of the balanced translocation at $3 \mathrm{q} 23$ or $10 \mathrm{q} 11.2$. A map of the 13q deletion interval is shown in Fig. 4. It was striking that both deleted segments occurred in gene poor regions, so that, although a total of about $10 \mathrm{Mb}$ of DNA had been deleted, only six genes have been identified in the deleted region. Table 1 lists these genes, with what is known of their function. There were another 16 intervals of altered copy number ranging in size from $130 \mathrm{~kb}$ to $1.7 \mathrm{Mb}$, which is consistent with a "normal" degree of largescale copy number variation. One such variant was a duplication that included exon 1 of the PARKIN gene at chromosome 6q26 (162,549,892 to $162,723,034 \mathrm{bp})$. Because partial alter-

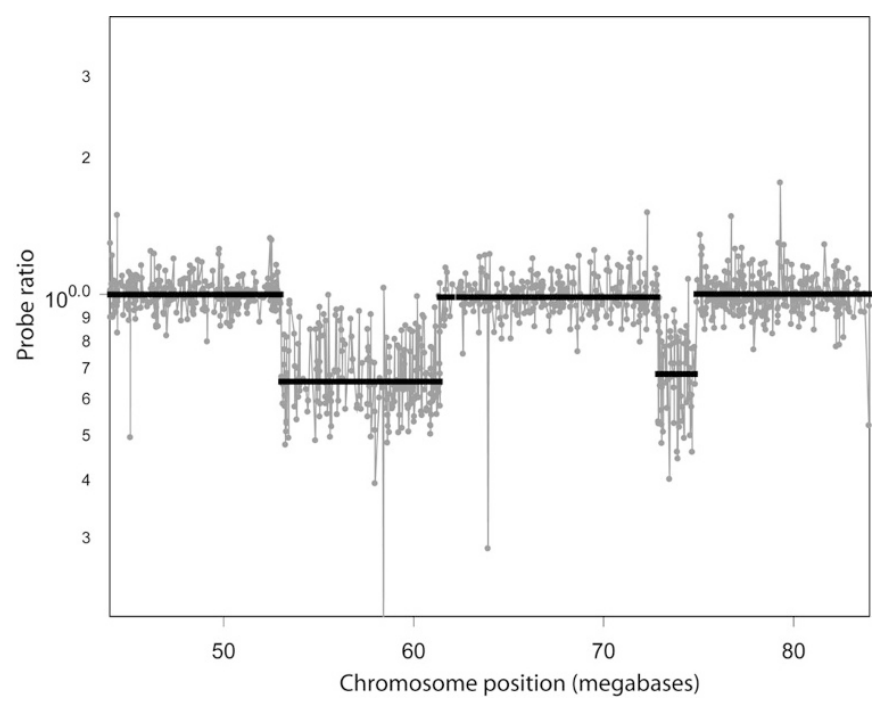

Fig. 3. ROMA scan results on chromosome 13 for Patient 1, after HMM analysis to define segmental changes. Solid black line defines two 1:2 deleted regions in 13q, with an intervening nondeleted region. ations in dosage of PARKIN have been associated with neurological disease, this lesion was further investigated by FISH.

\section{Patient 2}

ROMA detected a large deletion of chromosome 4 (427 probes, 58.8 to $81.9 \mathrm{Mb}$ ). Thus, the deletion was approximately $23 \mathrm{Mb}$ in length (Fig. 5), although it had been missed at the time of prenatal diagnosis.

\section{Patient 3}

ROMA detected a deletion of 22q, extending from approximately $43 \mathrm{Mb}$ to the telomere, and a duplication of $16 \mathrm{q}$ extending from $82 \mathrm{Mb}$ to the telomere. The deletion thus includes about $6 \mathrm{Mb}$ of $22 \mathrm{q}$ and the duplication extends through about $8 \mathrm{Mb}$ (Fig. 6).

\section{Confirmation of ROMA using FISH}

Table 2 and Figs. 7, 8, 9 show the results of the FISH experiments in Patient 1 . They confirmed the noncontiguous nature of the 13q deletion, as well as the amplification in $6 \mathrm{q}$ in the proband. The parents both showed a normal pattern for the chromosome 13 probes. The mother, but not the father, also showed the same amplification on $6 \mathrm{q}$ that was found in the proband.

\section{DISCUSSION}

For Patient 2, the ROMA analysis confirmed that the breakpoints were correctly assigned by conventional cytogenetics. There had been concern as to whether the deletion might involve the KIT oncogene at 4q12. ROMA analysis showed that the proximal breakpoint at $58.8 \mathrm{Mb}$ was distal to the KIT gene at $55.5 \mathrm{Mb}$. The sodium bicarbonate cotransporter, SLC4A4, is contained within the patient's deletion and is likely responsible for his proximal renal tubular acidosis. ${ }^{6}$ Also deleted are the

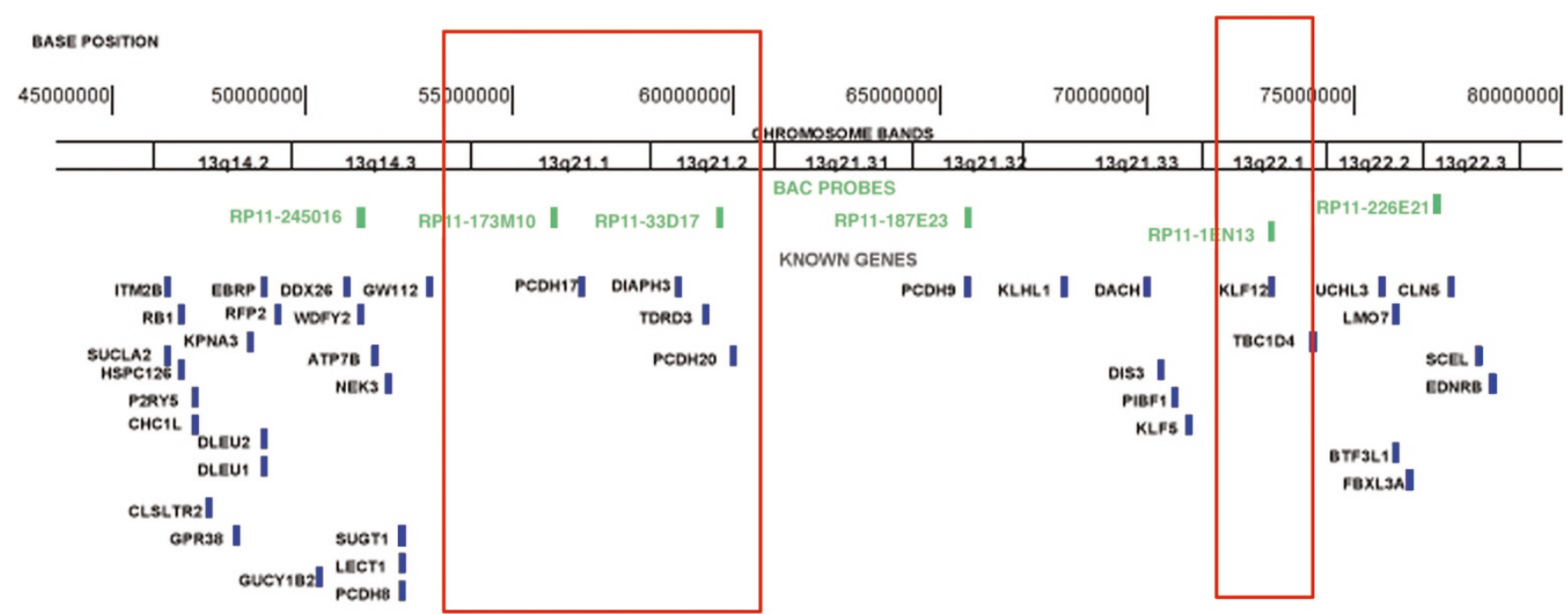

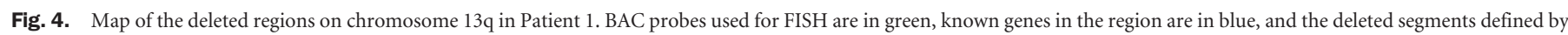
ROMA are outlined in red. 
Table 1

Genes deleted on chromosome 13 in Patient 1 (UCSC April 2003 freeze)

\begin{tabular}{|c|c|c|}
\hline Gene symbol & Gene name & Gene function \\
\hline PCDH17 & protocadherin 17 & Establishment and function of specific cell-cell connections in the brain \\
\hline DIAPH3 & diaphanous homolog 3 (Drosophila) & $\begin{array}{l}\text { Binds to GTP-bound form of Rho and to profilin; acts in a Rho-dependent } \\
\text { manner to recruit profilin to the membrane, where it promotes actin } \\
\text { polymerization; required for cytokinesis, stress fiber formation, and } \\
\text { transcriptional activation of the serum response factor }\end{array}$ \\
\hline TDRD3 & tudor domain containing 3 & Function not known \\
\hline $\mathrm{PCDH} 20$ & protocadherin 20 & Belongs to the protocadherin gene family; similar function to protocadherin 17 \\
\hline KLF12 & Kruppel-like factor 12 & $\begin{array}{l}\text { Confers strong transcriptional repression to the } \mathrm{AP}-2 \text { alpha gene; binds to a } \\
\text { regulatory element in the AP-2alpha gene promoter }\end{array}$ \\
\hline TBC1D4 & TBC1 domain family member 4 & May act as a GTPase activating protein for Rab family protein(s) \\
\hline
\end{tabular}

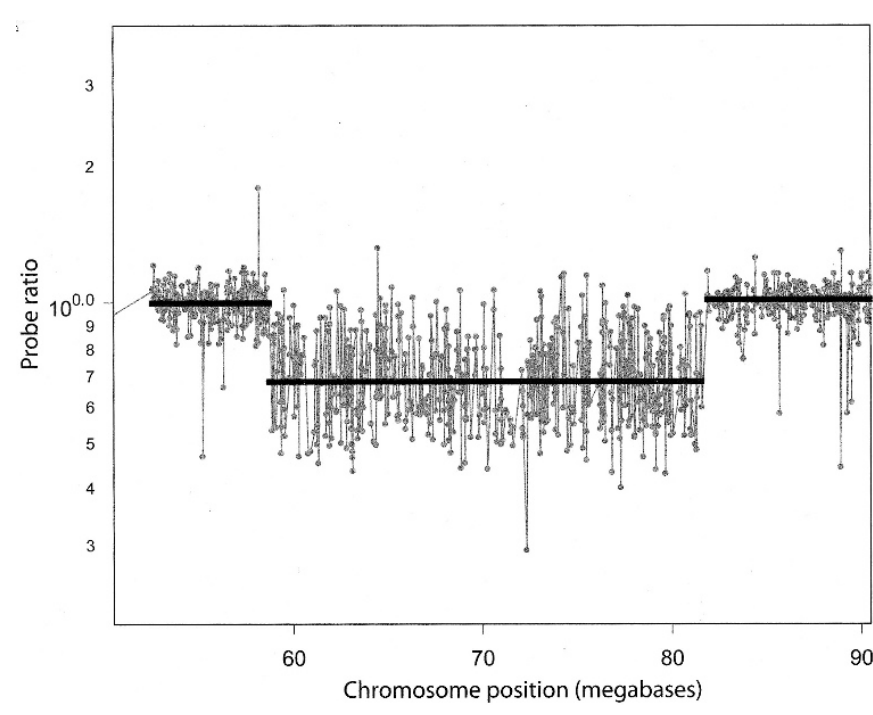

Fig. 5. ROMA scan results on chromosome 4 for Patient 2, after HMM analysis. Solid black line defines the large 1:2 deletion in $4 \mathrm{q}$.

CXCL 1, 2, 3, 5, 6, 9, 10, and 11 members of the chemokine family of cytokines, which may be responsible for his recurrent infections. The deleted interval does not contain any genes known to be involved in glucose metabolism that might explain his recurrent hypoglycemia.

For Patient 3, ROMA analysis demonstrated that, even though the imbalance could be detected only by FISH with subtelomeric probes, the deleted and amplified chromosomal segments were both $>5 \mathrm{Mb}$ and occurred in gene-rich regions. This case exemplifies the fact that terminal rearrangements detected by subtelomeric FISH may involve fairly large chromosomal segments not distinguishable by G-banding and do not necessarily involve only the most terminal regions. Application of ROMA to other "subtelomeric rearrangements" could allow the identification of "hotspots" for such exchanges and improve the ability to define new clinical syndromes.

For Patient 1, the ROMA analysis was extremely informative, revealing previously unknown complexity of the deletion and providing an explanation for the minimal clinical abnormalities found in this patient. Interstitial deletions of chromo- some 13 involving bands q14.3 to q21 have been previously reported and often have a relatively mild phenotype, involving mild to moderate mental retardation, variable minor anomalies, and growth retardation. ${ }^{7,8}$ One familial deletion of only q21 is reported 9 in a mother and daughter who were described as completely normal. The authors, in 1985, suggested that this might be a gene-poor region based on the Giemsa banding and replication pattern, which suggested it was mostly heterochromatin.

Dean et al. ${ }^{10}$ reviewed five cases in which a deletion of chromosome 13 was described to include band q21 and all or most of q22. All of these cases presented with mental retardation that was sometimes severe, delayed language acquisition, postnatal growth retardation, short hands, low set or posteriorly rotated ears, and a broad nasal bridge. Two patients had swallowing difficulties in early life similar to our patient. It is likely that the more severe phenotype in these cases is due to the deletion in band q22.

In our case, the parents had been advised that their child would likely have developmental delay, based on the discovery of a cytogenetically visible chromosome 13 deletion in a 3-month-old child who was hypotonic and growth retarded. The possible significance of the de novo balanced translocation was also discussed with the family. However, at the age of 3 years, his development is almost completely normal except for some delay in gross motor skills. Height and weight are at the 25th percentile, and there are no dysmorphic features except for cupped ears.

The complete DNA sequence and analysis of human chromosome 13 has recently been published. ${ }^{11}$ Of note in this chromosome is the low gene density, especially in two central regions from 52.9 to $90.7 \mathrm{Mb}$, corresponding to bands q21 and q31. The ROMA scan provides an explanation for why our patient with a deletion of approximately $10 \mathrm{Mb}$ is mildly affected. The two deletions in our patient (53 to $61.4 \mathrm{Mb}$ and from 72.8 to $74.8 \mathrm{Mb}$ ) occur in a "gene desert" on chromosome 13; the first deletion includes a 3-Mb region with no known genes. Only six genes have been identified in the region deleted in our patient. Deletions or mutations of these genes have not been reported and therefore it is not possible to correlate any 

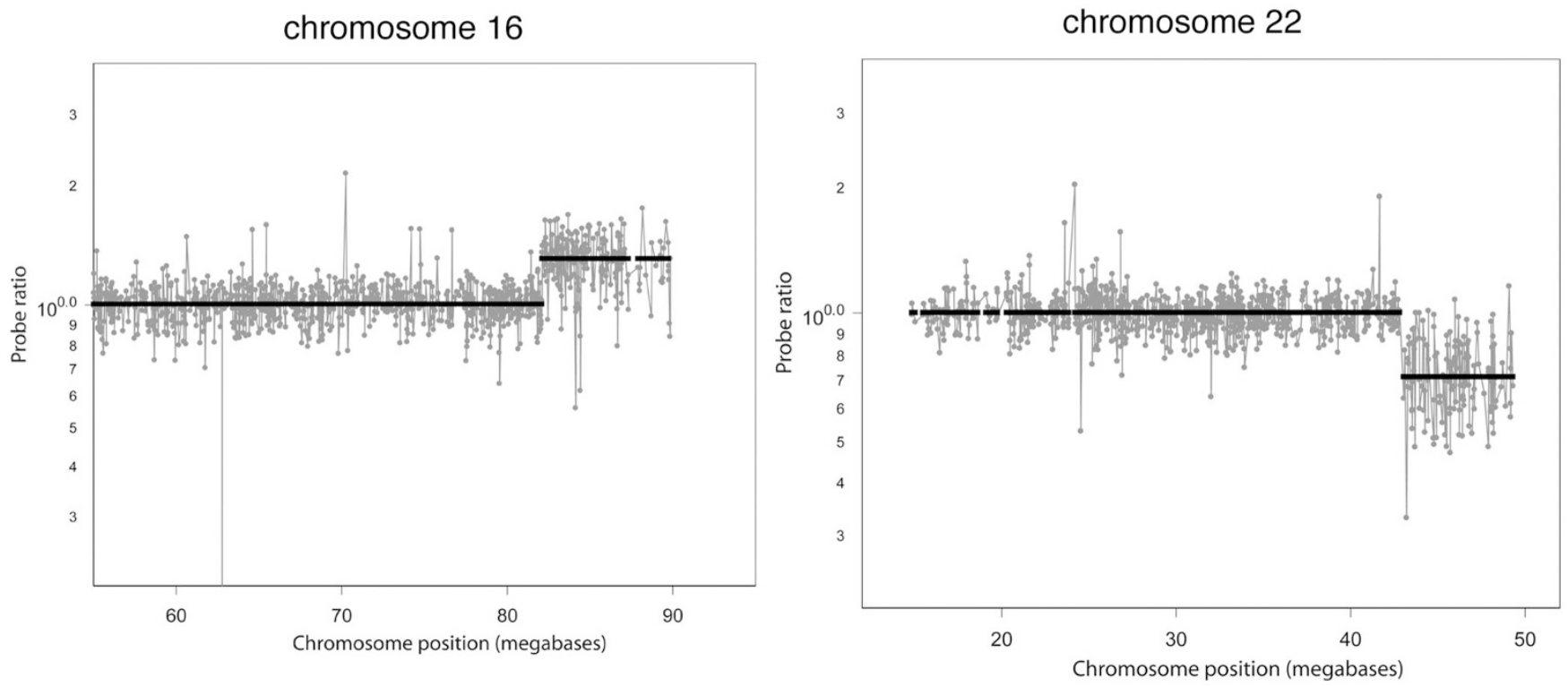

Fig. 6. ROMA scan results for chromosomes 16 and 22 for Patient 3 after HMM analysis. Solid black line defines the 3:2 duplication of 16q and the 1:2 deletion of 22q.

Table 2

FISH results with BAC/PAC clones on Patient 1

\begin{tabular}{|c|c|c|c|c|c|}
\hline \multirow{2}{*}{$\begin{array}{l}\mathrm{BAC} / \mathrm{PAC} \\
\text { clone }\end{array}$} & \multirow{2}{*}{$\begin{array}{l}\text { Chromosome } \\
\text { band }\end{array}$} & \multirow[b]{2}{*}{ Base position $^{a}$} & \multicolumn{3}{|c|}{ FISH signal Present $(+)$ or Absent $(-)$} \\
\hline & & & Patient & Mother & Father \\
\hline RP11-245D16 & $13 q 14.3$ & $51875570-51991999$ & + & + & + \\
\hline RP11-173M10 & $13 \mathrm{q} 21.1$ & $56074480-56231507$ & - & + & + \\
\hline RP11-307D17 & $13 \mathrm{q} 21.2$ & $59999802-60003998$ & - & + & + \\
\hline RP11-187E23 & $13 q 21.32$ & $66131615-66302973$ & + & + & + \\
\hline RP11-138N13 & $13 q 22.1-13 q 22.2$ & $74350431-74497138$ & - & + & + \\
\hline RP11-226E21 & $13 q 22.2-13 q 31.1$ & $76561670-76659146$ & + & + & + \\
\hline RP1-292F10 & $6 \mathrm{q} 26$ & 162601194-162717177 & $++^{b}$ & $++^{b}$ & + \\
\hline
\end{tabular}

${ }^{a}$ From the UCSC Genome Browser (April 2003 freeze).

${ }^{b}$ Amplified signal.

predicted deleterious effects of the deletion with the phenotype of the present case. Although the deletion in chromosome 13 had been shown not to include the RB1 gene by FISH, the parents had been told that it was possible that a breakpoint close to the gene might affect its function and had been advised to undergo biannual examination for retinoblastoma and osteosarcoma. The ROMA analysis determined that the deletion began at least $8 \mathrm{Mb}$ downstream of $\mathrm{RB}$, making it highly unlikely to affect the function of this gene.

The ROMA scan also detected no deletions or amplifications at the site of the translocation breakpoints in chromosomes $3 \mathrm{q} 23$ and 10q11.2. However, this does not preclude a breakpoint that interrupts a gene or interferes with gene expression.

The discovery of a noncontiguous deletion was a surprise, although we will not know how often this may occur until many more cases of interstitial deletions are examined with similar methods. Yu et al. ${ }^{12}$ report a noncontiguous deletion in a derivative chromosome, $\operatorname{der}(1) \mathrm{t}(1 ; 1)(\mathrm{p} 36 ; \mathrm{q} 44)$ (their Case 9), identified by microarray CGH. One explanation for this phenomenon would be an inversion in a parental chromosome, which would bring the noncontiguous deleted segments in juxtaposition before the deletion occurred. The presence of an inversion might even predispose to deletion at meiosis in an inversion heterozygote. Another explanation would be an error in the public database. In our case, FISH analysis confirmed the order of the BAC probes and did not demonstrate any inversion in the parental chromosomes involving the deleted segments of chromosome 13. However, such an inversion may still have been present in a parental germ cell.

PARKIN mutations and deletions have been described in patients with early-onset Parkinson disease, ${ }^{13,14}$ and there are also two reported cases of Parkinson disease with heterozygous duplications of exon $4 .{ }^{15}$ However, the presence of the PAR- 

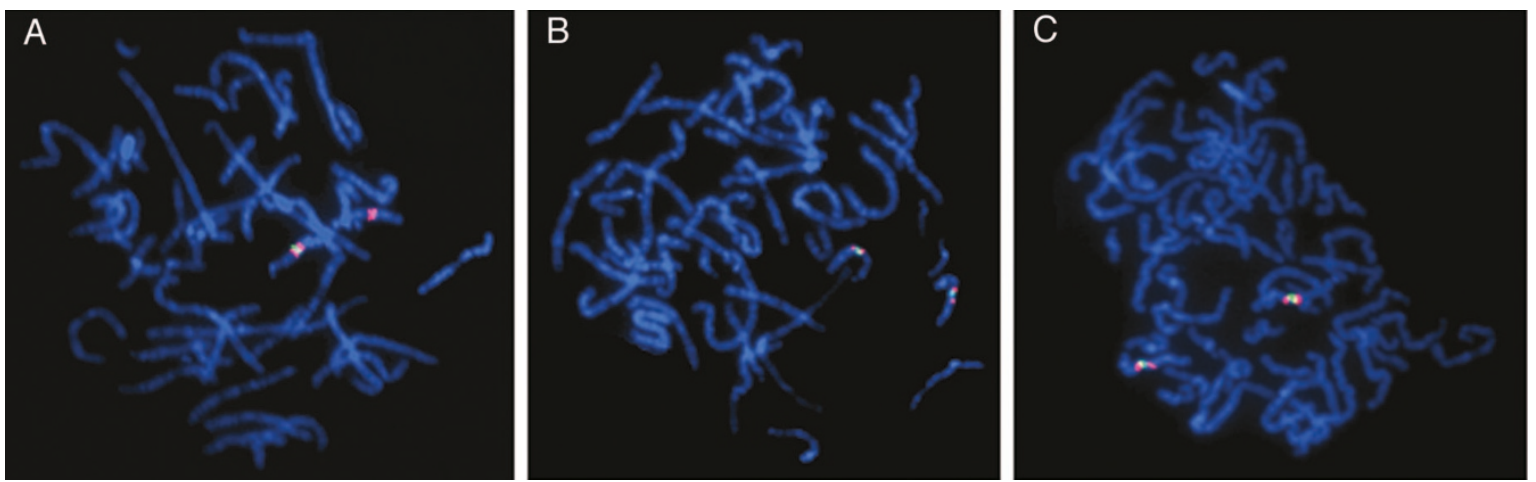

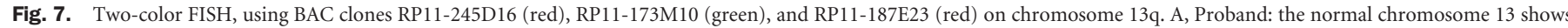

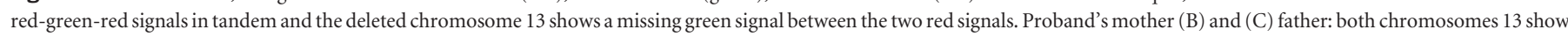
red-green-red signals in tandem.
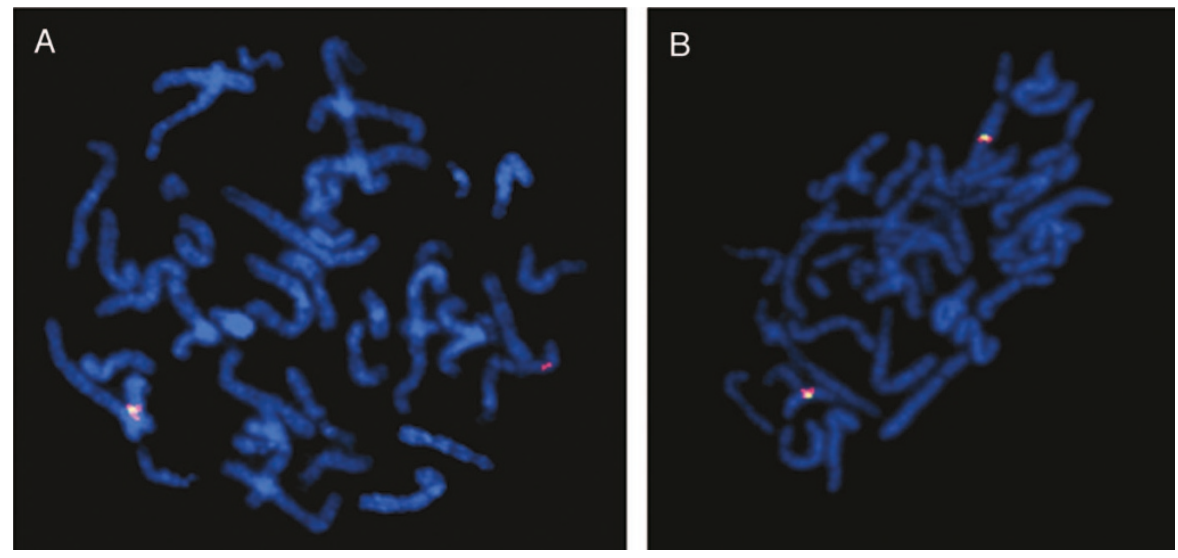

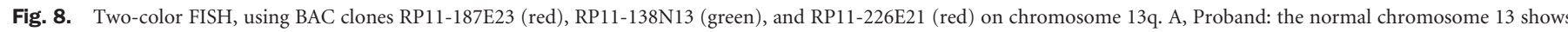

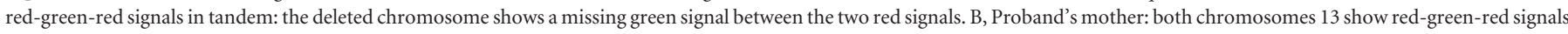
in tandem.

KIN duplication at $6 \mathrm{q} 26$ in both the child and the normal mother suggests that this change may not be relevant to the phenotype of Patient 1. Because analysis using the $85 \mathrm{~K}$ BglII II ROMA chip detects an average of 11 normal CNPs between unrelated individuals, ${ }^{5}$ most of the smaller-sized copy number differences detected by ROMA will be structural polymorphisms present in the human population. Knowledge of normal copy number polymorphisms will be essential for interpretation of genomic microarray data in clinical studies.

These cases illustrate the detailed information on cytogenetic abnormalities that is possible using the ROMA methodology. This method has major advantages over BAC microarray systems: resolution at least 3-fold higher than even a complete BAC tiling path; higher signal to noise ratio, due to the reduced complexity of the DNA and the lack of repetitive DNA in the small target oligonucleotides; flexible chip design; and the powerful method of statistical analysis to detect meaningful changes. ${ }^{16}$

At the present time, costs and limited availability limit the application of this technique. New technical innovations under development will reduce costs and make ROMA more widely available. It is likely that the future of cytogenetic anal- ysis will routinely involve the ability to define chromosomal aberrations at this "ultra" high level of resolution. Together with the information from the human genome sequence, this should immensely improve our ability both to understand the mechanisms of chromosomal rearrangements and to provide more accurate prognosis both prenatally and postnatally to parents of offspring with chromosomal aberrations.

\section{ACKNOWLEDGMENTS}

This work was supported by grants to M.W. from the National Institutes of Health and NCI (CA81674; CA078544; HG02606) and The Simons Foundation. M.W. is an American Cancer Society Research Professor. J.S. was supported by an NIH Training Grant (5T32 CA069311-25). We thank Eriny Thoroffsdotir and KristinAnna Olafsdottir of LindgGen EHF for providing technical support.

\section{References}

1. Ishkanian AS, Malloff C, Watson SK, deLeeuw R, Chi B, Coe B, Snijders A et al. A tiling resolution DNA microarray with complete coverage of the human genome. Nat Genet 2004;36:299-303. 

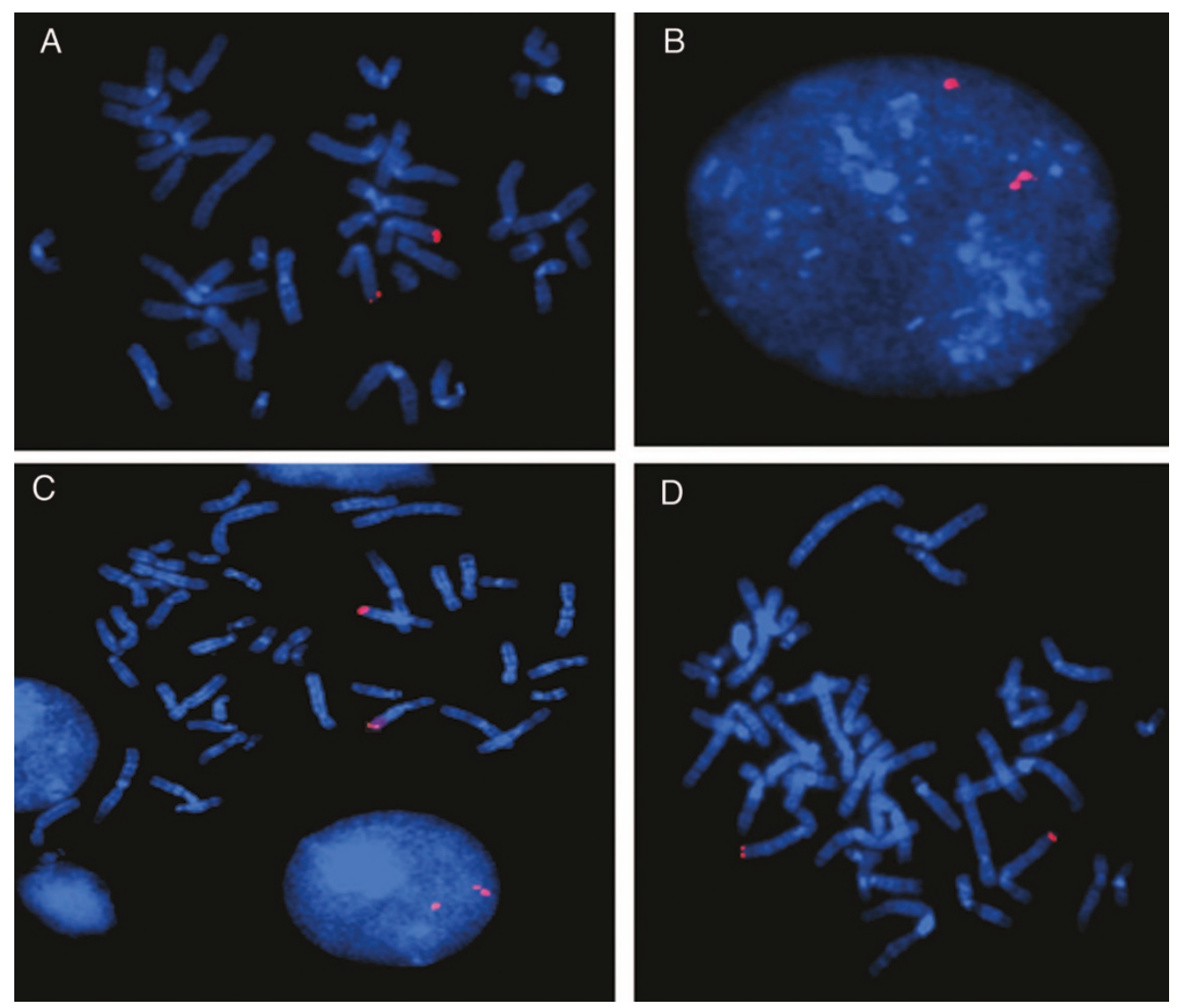

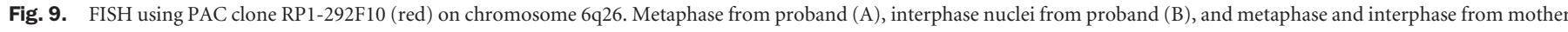

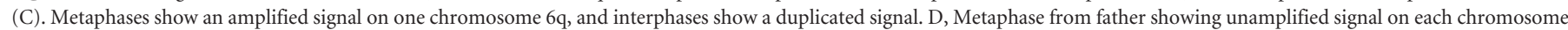
6.

2. Shaw-Smith C, Redon R, Rickman L, Rio M, Willatt L, Fiegler H et al. Microarray based comparative genomic hybridization (array-CGH) detects submicroscopic chromosomal deletions and duplications in patients with learning disability/mental retardation and dysmorphic features. J Med Genet 2004;41:241-248.

3. Lucito R, Healy J, Alexander J, Reiner A, Esposito D, Chi M et al. Representational oligonucleotide microarray analysis: a high-resolution method to detect genome copy number variation. Genome Res 2003;10:2291-2305.

4. Healy J, Thomas EE, Schwartz JT, Wigler M. Annotating large genomes with exact word matches. Genome Res 2003;10:2306-2315.

5. Sebat J, Muthuswamy T, Troge J, Alexander J, Young J, Lundin P et al. Gene copy number polymorphism is common in the human population and widely distributed in the genome. Science 2004;305:525-528.

6. Igarashi T, Sekine T, Inatomi J, Seki G. Unraveling the molecular pathogenesis of isolated proximal renal tubular acidosis. J Am Soc Nephrol 2002;2171-2177.

7. Brown S, Gersen S, Anyane-Yeboa K, Warburton D. Preliminary definition of a "critical region" of chromosome 13 in q32: report of 14 cases with 13 q deletions and review of the literature. Am J Med Genet 1993;45:52-59.

8. Nielsen J, Homma A, Christiansen F, Rasmussen K, Saldana-Garcia P. Deletion long arm 13. Hum Genet 1977;37:339-345.

9. Couturier J, Morichon-Delvallez N, Dutrillaux B. Deletion of band 13q21 is compatible with normal phenotype. Hum Genet 1985;70:87-91.
10. Dean JC, Simpson S, Couzin DA, Stephen GS. Interstitial deletion of chromosome 13: prognosis and adult phenotype. J Med Genet 1991;8:533-535.

11. Dunham A, Matthews LH, Burton J et al. The DNA sequence and analysis of human chromosome 13. Nature 2004;428:522-528.

12. Yu W, Ballif BC, Kashork CD et al. Development of a comparative genomic hybridization microarray and demonstration of its utility with 25 well-characterized 1p36 deletions. Hum Mol Genet 2003;12:2145-2152.

13. Abbas N, Lucking CB, Ricard S, Durr A, Bonifati V, De Michele G, Bouley S et al. A wide variety of mutations in the parkin gene are responsible for autosomal recessive parkinsonism in Europe. French Parkinson's Disease Genetics Study Group and the European Consortium on Genetic Susceptibility in Parkinson's Disease. Hum Mo Genet 2001;4:567-574.

14. Lucking CB, Durr A, Bonifati V, Vaughan J, De Michele G, Gasser T et al. Association between early-onset Parkinson's disease and mutations in the parkin gene. French Parkinson's Disease Genetics Study Group. N Engl J Med 2000;342:1560 1567.

15. Hedrich K, Mann M, Lanthaler AJ, Dalski A, Eskelson C, Landt O et al. The importance of gene dosage studies: mutational analysis of the parkin gene in early-onset parkinsonism. Hum Mol Genet 2001;10:1649-1656.

16. Mantipragada KK, Buckley PG, Diaz de Stahl T, Dumanski JP. Genomic microarrays in the spotlight. Trends Genet 2004;20:87-94. 